\title{
Characterization Of Periodontal Disease in A Population of Patients With Diabetes Mellitus
}

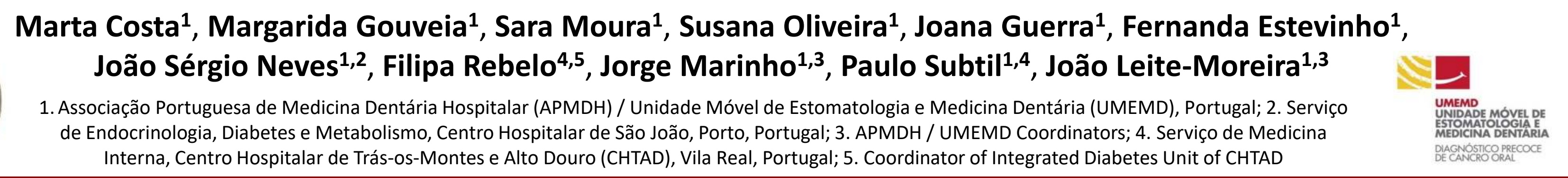

INTRODUCTION

Diabetes mellitus (DM) and periodontal disease (PD) represent two pathological entities with high prevalence. Several studies suggest a bidirectional relationship between these two diseases. The evaluation of the incidence of PD in a population of patients with DM is determinant to define strategies of prevention and treatment.

\section{METHOdS AND RESULTS}

\section{MetHODS}

We evaluated 179 consecutive patients followed in the diabetes outpatient clinic of Trás-os-Montes and Alto Douro Hospital Center (CHTMAD), Vila Real, Portugal.

The population was evaluated for demographic characteristics, anthropometric parameters, HbA1c, DM complications, comorbidities and pharmacological therapy.

Oral health habits were also characterized and an evaluation of the oral cavity was carried out by a team of medical dentists with evaluation of depth of periodontal pockets, gingival recession, and bleeding on probing

The presence and severity of PD was characterized by the CPI (Community Periodontal Index): 0 (healthy periodontal condition), 1 (gingival hemorrhage), 2 (calculus and bleeding), 3 (shallow periodontal pockets - 4 to $5 \mathrm{~mm}$ ), 4 (deep periodontal pockets - $\geq 6 \mathrm{~mm}$ )

\begin{tabular}{|c|c|}
\hline Sex & \\
\hline Female, \% & $45.8 \%$ \\
\hline Male, $\%$ & $54.2 \%$ \\
\hline Age, years & $62.9 \pm 12.0$ \\
\hline \multicolumn{2}{|l|}{ Education } \\
\hline$<4$ th year & $12.4 \%$ \\
\hline 4th year & $59.6 \%$ \\
\hline 9th year & $14.3 \%$ \\
\hline 12 th year & $6.8 \%$ \\
\hline High education & $6.8 \%$ \\
\hline $\mathrm{BMI}, \mathrm{kg} / \mathrm{m}^{2}$ & $31.8 \pm 6.8$ \\
\hline Abdominal circumference, $\mathrm{cm}$ & $104.2 \pm 13.5$ \\
\hline \multicolumn{2}{|l|}{ Type of Diabetes } \\
\hline Type 2, \% & $94.4 \%$ \\
\hline Type 1, \% & $5.0 \%$ \\
\hline Others, \% & $0.6 \%$ \\
\hline
\end{tabular}

\section{RESULTS}
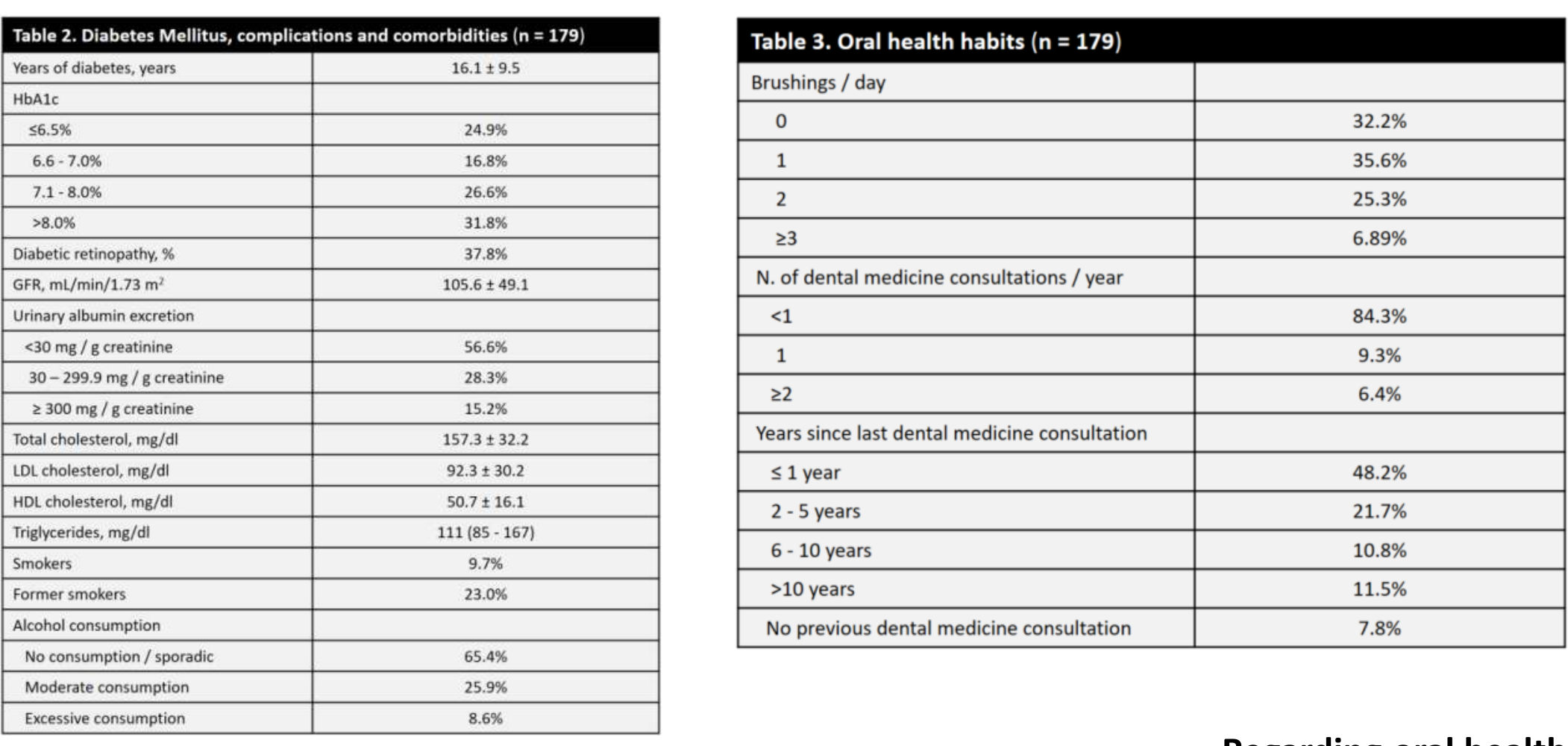

\begin{tabular}{|c|c|c|c|}
\hline & CPI 0-2 (n=18) & CPI 3-4 (n=132) & P value \\
\hline Male sex, \% & $39.4 \%$ & $60.6 \%$ & 0.008 \\
\hline Age, years & 62.5 & 61.6 & 0.652 \\
\hline Education $<9$ th year, $\%$ & $64.7 \%$ & $70.7 \%$ & 0.615 \\
\hline $\mathrm{BMI}, \mathrm{kg} / \mathrm{m}^{2}$ & $33.2 \pm 2.2$ & $31.7 \pm 0.7$ & 0.452 \\
\hline Type 2 Diabetes, \% & $94.4 \%$ & $93.1 \%$ & 0.929 \\
\hline Years of diabetes, years & $15.1 \pm 2.8$ & $15.7 \pm 0.9$ & 0.820 \\
\hline HbA1c, \% & $7.53 \pm 0.30$ & $8.21 \pm 0.57$ & 0.653 \\
\hline Diabetic retinopathy, \% & $16.7 \%$ & $42.4 \%$ & 0.088 \\
\hline Diabetic nephropathy, \% & $62.5 \%$ & $65.0 \%$ & 0.844 \\
\hline Smokers and former smokers, \% & $16.7 \%$ & $34.7 \%$ & 0.127 \\
\hline Excessive alcohol consumption & $5.6 \%$ & $10.2 \%$ & 0.535 \\
\hline Brushings / day, $\mathrm{n}$ & $1.6 \pm 0.2$ & $1.0 \pm 0.1$ & 0.006 \\
\hline 21 dental medicine consultations / year, $\%$ & $27.8 \%$ & $15.0 \%$ & 0.171 \\
\hline Years since last consultation, years & $2.1 \pm 0.7$ & $3.8 \pm 0.7$ & 0.356 \\
\hline Bad oral hygiene, \% & $50.0 \%$ & $72.1 \%$ & 0.026 \\
\hline
\end{tabular}

\begin{tabular}{|l|l|}
\hline \multicolumn{2}{|l|}{ Table 4. Objective evaluation of oral health and CPI classification ( $\mathbf{n}=179)$} \\
\hline Oral health & \\
\hline Good & $3.5 \%$ \\
\hline Moderate & $32.4 \%$ \\
\hline Bad & $64.2 \%$ \\
\hline CPI classification \\
\hline 0 - healthy periodontal condition & \\
\hline 1 - gingival hemorrhage & $2.2 \%$ \\
\hline 2 - calculus and bleeding & $3.9 \%$ \\
\hline 3 - shallow periodontal pockets & $3.9 \%$ \\
\hline 4- deep periodontal pockets & $35.8 \%$ \\
\hline x- edentulous & $38.0 \%$ \\
\hline
\end{tabular}

Regarding oral health habits, $32.2 \%$ of the subjects did not brush daily, $84.3 \%$ had $<1$ medical dentist consultation per year and $35.2 \%$ did not had any medical dentist consultation in the last 5 years.

97.3\% of the patients had PD (87.9\% with CPI classification 3-4).

The presence of more severe PD (CPI 3-4) was associated with male sex $(p=0.008)$, lower number of brushings per day $(p=0.006)$ and poor oral hygiene $(p=0.026)$.

Patients with more severe PD were also shown a tendency to a higher prevalence of diabetic retinopathy $(42.4 \%$ vs $16.7 \%, p=0.088)$, higher HbA1c $(8.21 \pm 0.57$ vs $7.53 \pm 0.30, P=0.653)$ and current or past smoking $(34.7 \%$ vs $16.7 \%, p=0.127)$.

\section{CONCLUSIONS}

We observed a high prevalence of PD in patients followed at the CHTMAD diabetes clinic. The presence of poor oral hygiene habits and the difficulty in accessing oral health care seem to contribute to this high prevalence. Given their frequency, screening and early treatment of PD should be a priority in patients with DM. 